\title{
Expertise and attunement to kinematic constraints
}

\begin{abstract}
Three experiments were undertaken to ascertain the extent to which expertise in natural anticipatory tasks is characterised by superior attunement to the biomechanical (kinematic) constraints of the movement pattern being observed. Twelve world-class and twelve nonexpert badminton players were required to predict the depth of an opponentôs stroke from either video displays or point-light displays of the opposing playerôs hitting action. The information available within the displays was manipulated through temporal and/or spatial occlusion. Consistent with predictions that can be derived from the constraint-attunement hypothesis (Vicente and Wang, 1998 Psychological Review 10533 ï 57), experts showed: (i) an unchanged pattern of information pick-up when the display was reduced from video to point-light and only kinematic information was available; (ii) superior information pick-up from kinematic features that non-experts could use; and (iii) attunement to early kinematic information from the lower body to which non-experts were not sensitive. Consistent with predictions that can be derived from a common-coding perspective (Prinz, 1997 European Journal of Cognitive Psychology 9129 ï 154), the anticipation of stroke depth was facilitated more for experts than non-experts when the perceptual display provided linked segment information reminiscent of the cross-segmental torque transfers that occur during expert movement production.
\end{abstract}

Keyword: Attunement; Kinematic; Motion perception; Motor skills; Movement; Distance perception 